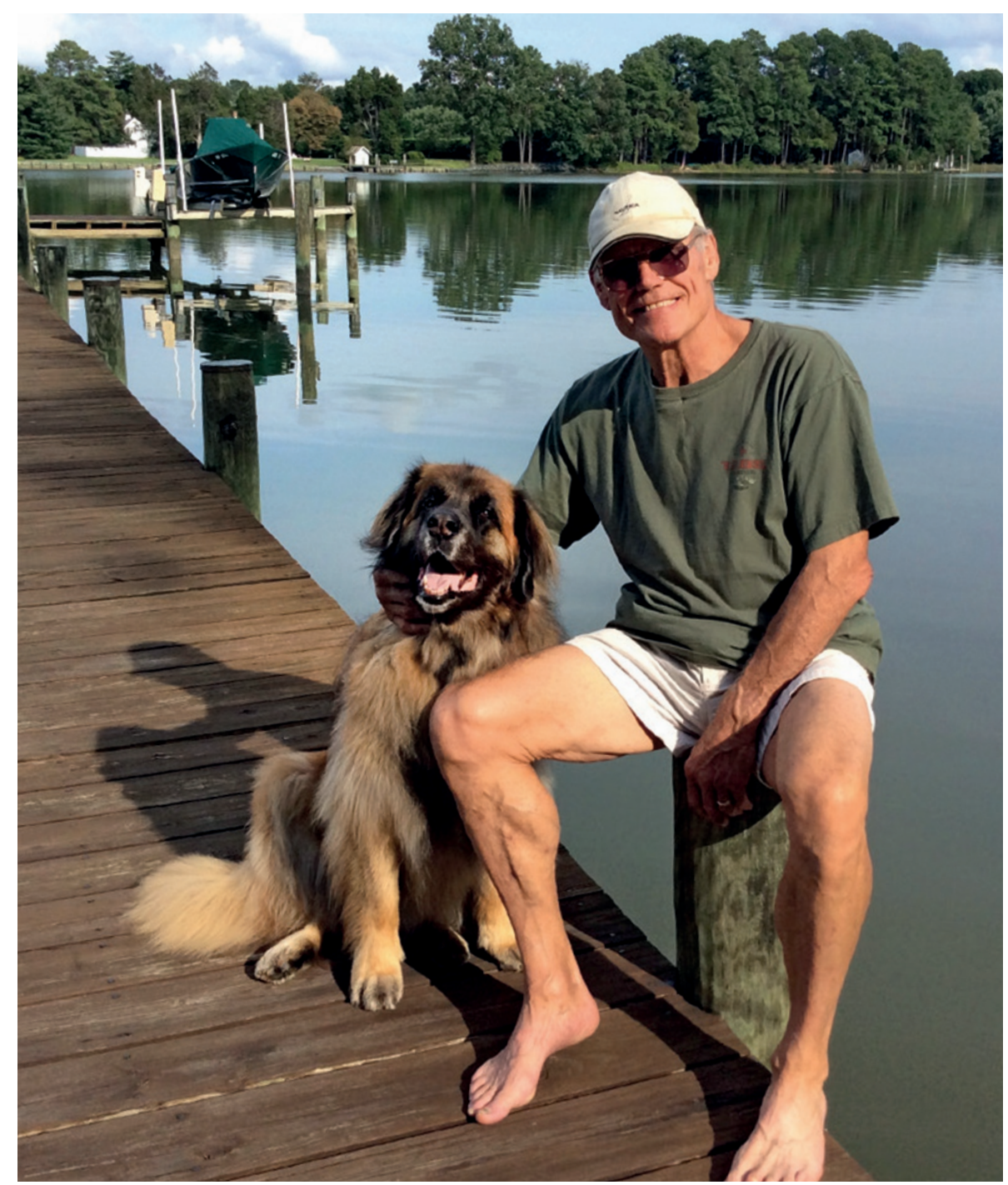

Having opted for active surveillance, Bill Wilson has avoided surgery and continues with his busy lifestyle.

TREATMENT

\title{
When less is more
}

\section{Surveillance is becoming a watchword for men with less-aggressive prostate cancer. If and when the disease progresses, new and newly-timed therapies are at hand.}

\section{BY MEREDITH WADMAN}

$\mathrm{W}$ hen Bill Wilson learned that he had prostate cancer in 2011, he wanted to race to the nearest operating theatre. Wilson, a 71-year-old former IBM executive from St. Michael's, Maryland, says his first thought was: "I'm going to get it out of there."

But his urologist encouraged him to talk to other specialists, and so Wilson visited Ballentine Carter, a prostate-cancer researcher never be; therefore, I did not have to immediately have aggressive treatment."

Aggressive treatment is common: according to a recent report ${ }^{1}, 50 \%$ of US men diagnosed with low-risk prostate cancer between 2010 and 2013 underwent radical prostatectomy (surgical removal of the prostate and surrounding lymph nodes). However, active surveillance is being offered by a growing number of physicians, and taken up by a rising number of men (see 'Active prime time'). A large study in Sweden, led by Stacy Loeb, a New York University urologist, found that nearly half of men diagnosed with low-risk disease between 1998 and 2011 opted for active surveillance, with the proportion increasing over this time period ${ }^{2}$. In the United States, the turn toward active surveillance for low-risk disease has been dramatic, growing from single-digit percentages in the late 1990s to $40 \%$ between 2010 and 2013 (ref. 1).

Disagreements remain on which cancers are best suited to active surveillance, and on how to monitor those men selected. Nonetheless, active surveillance "has spiked and become prime time", says Loeb. And for men whose disease does progress, new treatments and protocols are lengthening and improving the quality of their remaining life.

\section{A BALANCING ACT}

According to criteria set by Carter's Johns Hopkins group, Wilson was a good candidate for active surveillance: his tumour cells did not look aggressive under the microscope; the tumour could not be felt by digital rectal exam and was only picked up by needle biopsy; and he had relatively low levels of prostate-specific antigen (PSA), a blood marker that is a proxy for the presence of prostate cancer (Wilson underwent the biopsy only because his PSA was found to be slightly elevated: 0.57 nanograms per millilitre above the threshold of $4 \mathrm{ng} \mathrm{ml}^{-1}$.)

The active-surveillance regimen requires Wilson to visit Johns Hopkins every six months for a digital rectal exam and a blood test. Annually, he undergoes either a biopsy or a magnetic resonance imaging scan, for a more detailed inspection. His most recent biopsy worried Wilson slightly because small areas of cancer were found in 3 of the 12 tissue samples taken, up from 2 of the 12 at diagnosis. Still, the cancer cells did not appear more aggressive, and his PSA levels remained reassuringly low.

The surge in uptake of active surveillance is due, in part, to the response of a medical community that was roundly criticized for overtreatment of a disease that is too readily identified by PSA screening (see page S120). By offering active surveillance as a conservative way to manage men at lower risk, clinicians hope that the balance of harms and benefits from PSA screening will shift. "The acceptance of surveillance is going to be a crucial piece of rehabilitating PSA screening," 
$\rightarrow$.: says Laurence Klotz, a urologist in Sunnybrook

崖 Health Sciences Centre at the University of ن̈ Toronto, Canada.

\& Klotz's group has published some of the longest-term data on the results of active surveillance, which it began offering in 1995 (ref. 3). The Toronto group followed 993 men, most of whom had low-risk tumours. It also included some men with slightly higher-risk tumours who had other significant illnesses and less than ten years of life expectancy. The group was followed for a median of 6.4 years; $1.5 \%$ died of prostate cancer.

For men with low-risk tumours, the comparable outcome after surgical removal of the prostate is slightly better. A 2011 study involving 24,000 men showed that, after 15 years of follow-up, between $0.2 \%$ and $1.2 \%$ on average had died of prostate cancer ${ }^{4}$. But in the same study, men with slightly higher-risk tumours - equivalent to the riskier subgroup in the Toronto cohort — did not fare as well: their average 15 -year prostate-cancer-specific mortality ranged from $4.2 \%$ to $6.5 \%$.

Carter's team published its results in August $^{5}$. Of the 1,298 men with low- or verylow-risk disease placed on active surveillance since 1995 , only $0.1 \%$ had died of prostate cancer at 10 years of follow-up; at 15 years, the figure was unchanged.

"The take-home message is, in well-chosen patients, active surveillance is safe," says Fred Saad, a urologist at the University of Montreal Hospital Centre in Canada. However, the Johns Hopkins and Toronto studies also highlight one of the sticking points that activesurveillance advocates are still wrestling with: inclusion criteria. The Johns Hopkins group was more conservative than the Toronto team: in Baltimore, only low-risk men were enrolled, which could help to explain the better results. In a 2014

review of ten activesurveillance studies, the approach seemed to reduce overtreatment without compromising men's ten-year cancer sur-

"The take-home message is, in well-chosen patients, active surveillance is safe."

\section{ACTIVE PRIME TIME}

In US men diagnosed with low-risk prostate cancer, the number choosing active surveillance is rising.

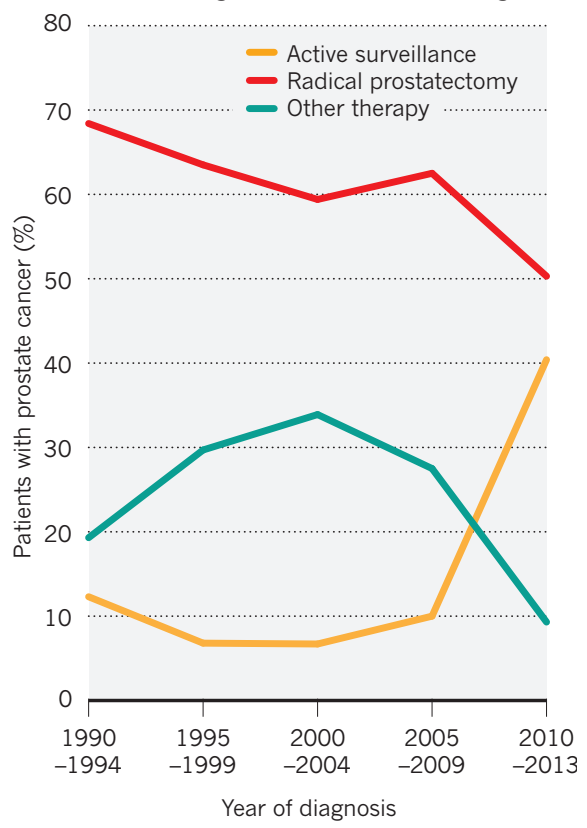

controlled trial in which 1,643 men newly diagnosed with localized prostate cancer are randomly assigned to receive active monitoring, surgery or radiation therapy. Hamdy's group plans to report results of the ProtecT trial, showing disease-specific survival rates at a median ten years' follow-up, as early as spring 2016.

\section{SECOND ACTS}

For men whose disease at diagnosis is too aggressive for active surveillance, treatment begins with some combination of surgery, radiation and medication - the first-line treatments for decades. Because prostatecancer cells are stimulated to grow by testosterone, more than $90 \%$ of which is made in the testes, one approach is surgical castration: the operative removal of the testes. (The rest of the body's testosterone is made in the adrenal glands.) The same end can be achieved with medical castration - the use of drugs that suppress the release of hormones that stimulate testosterone production.

Nearly all tumours eventually become resistant to these testosterone-lowering approaches, and when they spread, they are described as metastatic castration-resistant prostate cancer. For men in this stage, the past five years have seen remarkable advances in both new, life-prolonging drugs and in better ways to use older agents.

The drugs abiraterone and enzalutamide were first approved in 2011 and 2012, respectively. Abiraterone works primarily by interfering with testosterone synthesis, whereas enzalutamide prevents the hormone from binding to the androgen receptor. Although neither is curative, the advent of these oral agents has improved both survival and quality of life for men with advanced disease. "It is fantastic to give drugs that have almost no side effects and that make men feel better, have less pain and live longer," says Saad. Nonetheless, over time, most patients develop resistance to these drugs - a reality that has galvanized the hunt for new therapeutics (see page S128).

In another positive development, striking new evidence shows that a change in the timing of chemotherapy can buy men with metastatic disease many more months of life. Docetaxel - a chemotherapy agent that suppresses cancer-cell division - was approved in 2004 for use after medical or surgical castration has failed. But results of the CHAARTED trial published this year showed that the lives of men with metastatic disease were significantly extended when they were given docetaxel earlier, simultaneously with castration ${ }^{7}$. They lived a median of 14 months longer than men who underwent only medical or surgical castration. For men with the most extensive disease, the difference was still more pronounced: 17 months. The results "were really dramatic", says Matthew Cooperberg, a prostate-cancer specialist at the University of California, San Francisco. "You don't see a 17-month survival advantage very often in these types of trials."

The CHAARTED trial was led by Christopher Sweeney, a physician at the Dana-Farber Cancer Institute in Boston, Massachusetts. He suggests that the success came from deploying drugs with different mechanisms of action, thus targeting both the testosterone-sensitive and testosteroneinsensitive cancer cells simultaneously. "That's speculation, but something like that might be happening," says Sweeney. He and others are now running trials to see whether patients will benefit when the newer drugs, enzalutamide and abiraterone, are likewise deployed alongside castration earlier in the course of metastatic disease.

Meanwhile, having been spared aggressive treatment, Wilson is taking the 'active' part of his active surveillance seriously. He spends his time sailing his 35-foot sloop, Adagio, training his dog Jeter for agility contests, and getting out into the countryside - his disease far from his mind. "I just came back from hiking in the mountains in Arizona and put the 40-year-olds to shame."

Meredith Wadman is a freelance writer based in Washington, DC, and an editorial fellow at New America.

1. Cooperberg, M. R. \& Carroll, P. R. J. Am. Med. Assoc. 314, 80-81 (2015).

2. Loeb, S. et al. J. Urology 190, 1742-1749 (2013).

3. Klotz, L. et al. J. Clin. Oncol. 33, 272-277 (2015).

4. Eggener, S. E. et al. J. Urology 185, 869-875 (2011)

5. Tosoian, J. J. et al. J. Clin. Oncol. http://dx.doi.org/ $10.1200 / J C 0.2015 .62 .5764$ (2015).

6. Thomsen, F. B. et al. J. Surg. Oncol. 109, 830-835 (2014).

7. Sweeney, C. J. et al. N. Engl. J. Med. 373, 737-746 (2015).
Hamdy hopes to shed new light on this question with a first-of-its-kind, randomized 